\title{
Fenologia reprodutiva, biologia floral e polinização de Allamanda blanchetii, uma Apocynaceae endêmica da Caatinga ${ }^{1}$
}

\author{
LENYNEVES DUARTE ALVINO DE ARAÚJO²,5, ZELMA GLEBYA MACIEL QUIRINO³ e \\ ISABEL CRISTINA MACHADO ${ }^{4}$
}

(recebido: 14 de junho de 2010; aceito: 05 de maio de 2011)

\begin{abstract}
Reproductive phenology, floral biology and pollination of Allamanda blanchetii, an Apocynaceae endemic of the Caatinga). The reproductive phenology, floral biology, pollination mechanism and breeding system of Allamanda blanchetii A.DC. were studied in natural populations in the Almas Farm, Paraíba, Brazil. Allamanda blanchetii is a shrubby species, endemic of the Caatinga, flowering from February to July, with peak in March-April. The fruiting followed the flowering and continued until September. The tubular flowers are pink, their strong herkogamy preventing self pollination. The pollen grains are deposited in the upper side of the style head (non-receptive region), still in the pre anthesis phase (dehiscent anther), characterizing a secondary pollen presentation. The flowers produce an average of $36.6 \mu \mathrm{L}$ of nectar, that is the only resource to the floral visitors. Allamanda blanchetii is self incompatible and the results suggest a late-acting self-incompatibility mechanism. The floral attributes are compatible with the melittophilous and psychophilous syndromes. In fact, the observed pollinators were the bee Eulaema nigrita Lepeletier, as main pollinator, and four species of butterflies, which act as secondary pollinators. The occurrence of strong herkogamy, secondary pollen presentation and self-incompatibility mechanism indicates high degree of specialization of pollination and breeding systems of $A$. blanchetii.
\end{abstract}

Key words - herkogamy, secondary pollen presentation, self incompatibility

RESUMO - (Fenologia reprodutiva, biologia floral e polinização de Allamanda blanchetii, uma Apocynaceae endêmica da Caatinga). A fenologia reprodutiva, a biologia floral, o mecanismo de polinização e o sistema reprodutivo de Allamanda blanchetii A.DC. foram analisados em populações naturais na Fazenda Almas, Paraíba, Brasil. Allamanda blanchetii é uma espécie arbustiva, endêmica da Caatinga, com floração de fevereiro a julho e pico nos meses de março e abril. A frutificação acompanhou a floração e estendeu-se até o mês de setembro. As flores são tubulares e de coloração rosa, cuja forte hercogamia evita a autopolinização. O pólen é depositado na parte superior da cabeça do estilete (região não receptiva), ainda na fase de botão em pré antese (anteras deiscentes), caracterizando a apresentação secundária de pólen. As flores produzem uma média de $36,6 \mu \mathrm{L}$ de néctar, o qual é o único recurso para os visitantes florais. Allamanda blanchetii é auto-incompatível e os resultados sugerem mecanismo de incompatibilidade de ação tardia. Seus atributos florais estão relacionados com as síndromes de melitofilia e psicofilia e, de fato, os polinizadores registrados foram a abelha Eulaema nigrita Lepeletier como principal polinizador e quatro espécies de borboletas que atuam como polinizadores secundários. A ocorrência de forte hercogamia, apresentação secundária de pólen e mecanismo de auto-incompatibilidade indicam o elevado grau de especialização dos sistemas de polinização e reprodução de $A$. blanchetii.

Palavras-chave - apresentação secundária de pólen, auto-incompatibilidade, hercogamia

\section{Introdução}

A Caatinga compreende a quarta maior formação vegetacional do Brasil (Aguiar et al. 2002), cobrindo cerca de $800.000 \mathrm{~km}^{2}$ do território brasileiro (MMA 2002). Mesmo com toda a sua exclusividade global, a

1. Parte da dissertação de mestrado do primeiro autor, Programa de Pós-Graduação em Biologia Vegetal da Universidade Federal de Pernambuco, 50372-970 Recife, PE, Brasil.

2. Universidade Federal da Paraíba, Centro de Ciências Agrárias, Departamento de Ciências Biológicas, 58397-000 Areia, PB, Brasil.

3. Universidade Federal da Paraíba, Centro de Ciências Aplicada e Educação, Departamento de Engenharia Meio Ambiente, 58297-000 Rio Tinto, PB, Brasil.

4. Universidade Federal de Pernambuco, Centro de Ciências Biológicas, Departamento de Botánica, 50372-970 Recife, PE, Brasil.

5. Autor para correspondência: lenyy_duarte@yahoo.com.br/ lenyneves@cca.ufpb.br
Caatinga ainda vem sendo muito negligenciada(Velloso et al. 2002) e relativamente pouco estudada, especialmente no que se refere aos processos de polinização e sistema reprodutivo de suas espécies vegetais (Machado \& Lopes 2004).

A família Apocynaceae sensu lato é constituída por cerca de 424 gêneros distribuídos em cinco subfamílias difundidas nas regiões tropicais e subtropicais (Endress \& Bruyns 2000). Allamanda L. é um pequeno gênero dessa família compreendendo apenas 14 espécies nativas do continente americano (Sakane \& Shepherd 1986), sendo $A$. blanchetii A.DC. uma das cinco espécies de Apocynaceae endêmicas da Caatinga, ocorrendo preferencialmente nos afloramentos rochosos e distribuindo-se por todo o nordeste brasileiro (Giulietti et al. 2002).

As flores de Apocynaceae são relatadas como complexas e com mecanismos de polinização bastante 
especializados, tais como forte hercogamia e apresentação secundária de pólen (Schick 1980, 1982, Fallen 1985, 1986, Howell et al. 1993, Yeo 1993, Albers \& van der Maesen 1994, Lopes \& Machado 1999), o que aumenta a complexidade floral e o nível de especialização dos polinizadores. Os trabalhos já realizados referentes à ecologia da polinização para a família reportam as flores de Apocynaceae s.str. sendo polinizadas principalmente por abelhas (Herrera 1991, Albers \& van der Maesen 1994, Torres \& Galleto 1998, Lipow \& Wyatt 1999, Lopes \& Machado 1999, Koch et al. 2002, Tostes et al. 2003, Löhne et al. 2004, Raju et al. 2005) e lepidópteros (Waddington 1976, Haber 1984, Lin \& Bernadello 1999, Schlindwein et al. 2004, Darrault \& Schlindwein 2005), enquanto que a polinização por aves (Linhart \& Feinsinger 1980) e mamíferos (Faegri \& van der Pij1 1979) são mais raras na família. Não há registros de trabalhos descrevendo a biologia da polinização e o sistema reprodutivo para nenhuma espécie do gênero Allamanda.

Neste estudo, apresentamos dados sobre a fenologia reprodutiva, a biologia floral e o mecanismo da polinização, bem como os aspectos da biologia reprodutiva de Allamanda blanchetii, pretendendo responder as seguintes questões: as flores de $A$. blanchetii se enquadram em qual síndrome de polinização? Quais os polinizadores efetivos da espécie? Qual é o mecanismo reprodutivo apresentado por Allamanda blanchetii?

\section{Material e métodos}

Área de estudo-O trabalho de campo, com populações naturais de A. blanchetii, foi desenvolvido na Reserva Particular de Proteção da Natureza (RPPN) Fazenda Almas, localizada no Município de São José dos Cordeiros $\left(7^{\circ} 28^{\prime} 45^{\prime \prime} \mathrm{S}\right.$ e 36 54'18” W), Paraíba, Brasil. A RPPN, com 3.505 ha de área preservada, está incluída na Ecorregião da Depressão Sertaneja Setentrional, onde a vegetação local é caracterizada como Caatinga arbustiva a arbórea, sobre solos de origem cristalina (Velloso et al. 2002). A precipitação média anual fica em torno de 500 a $800 \mathrm{~mm}$, mas com extremos de até $350 \mathrm{~mm}$ ano $^{-1}$. Essa ecorregião compreende a área mais seca, o Cariri Paraibano, com temperaturas médias anuais elevadas entre 26 e $30^{\circ} \mathrm{C}$, e clima semi-árido quente (Velloso et al. 2002).

Uma exsicata de Allamanda blanchetii encontra-se depositada no Herbário UFP, Departamento de Botânica da UFPE, como espécime-testemunho: BRASIL. PARAíBA: RPPN Fazenda Almas, s.d., L.D.A. Araújo s.n. (UFP45.556).

Fenologia reprodutiva - A análise das fenofases reprodutivas foi realizada de outubro de 2006 a dezembro de 2007. Foram marcados 10 indivíduos para o acompanhamento fenológico quinzenal, estimando-se a intensidade de floração e frutificação, segundo Fournier (1974). O padrão fenológico foi analisado de acordo com Gentry (1974) e Newstrom et al. (1994) e o Índice de Sincronia de Floração foi calculado a partir de Augspurger (1983). Testes de Correlação de Spearman foram realizados para comparar a precipitação pluviométrica e a produção de flores, utilizando o programa BioEstat 3.0 (Ayres et al. 2003). Para evitar a utilização de clones, já que a espécie apresenta propagação vegetativa através de órgãos subterrâneos, os indivíduos foram marcados um em cada "ilha de vegetação", uma vez que a população estudada encontrava-se em uma área de inselbergs formada por pequenas subpopulações. Os dados de precipitação pluviométrica foram obtidos através da Agência Executiva de Gestão de Águas do Estado da Paraíba (Aesa).

Biologia floral - Flores e botões $(n=20)$ de diferentes indivíduos foram conservados em etanol, para a morfometria. Com auxílio de lamínula quadriculada sob microscópio óptico, a razão pólen/óvulo $(\mathrm{P} / \mathrm{O})$ foi estimada a partir da contagem direta do número de grãos de pólen por antera e de óvulos por botão floral (Cruden 1977), sendo os grãos, ao mesmo tempo, corados com solução a $2 \%$ de carmim acético para determinar a viabilidade polínica (Radford et al. 1974). Para este procedimento, foram utilizados botões em pré-antese $(n=10)$ cujas anteras já estavam deiscentes. Para estimar o número de grãos de pólen depositados na cabeça do estilete (apresentação secundária de pólen) foram retirados o cone de anteras juntamente com a cabeça do estilete de botões $(n=10)$ em pré-antese (com anteras deiscentes) e depositados sobre uma lâmina histológica. Em seguida, as anteras foram cuidadosamente separadas da cabeça do estilete e o pólen presente na parte superior da cabeça do estilete foi transferido para a mesma lâmina, tornando possível a contagem do número de grãos de pólen apresentados secundariamente.

Para verificar se ocorria nova deposição de grãos de pólen sobre a cabeça do estilete, após a flor ser bastante visitada no primeiro dia de antese, foram realizadas cinco simulações de visitas com fios de náilon $(0,2 \mathrm{~mm})$, sendo os mesmos posteriormente depositados em pequenos envelopes etiquetados, para posterior análise. No período restante da antese, as simulações foram repetidas até não mais se observar, com o auxílio de uma lupa, grãos de pólen nos fios de náilon. Em laboratório, os fios de náilon e as respectivas anteras e cabeça do estilete de cada flor submetida às simulações foram analisados quanto à presença de grãos de pólen.

Em campo, foi verificado o recurso disponível para os visitantes florais, o volume e a concentração de açúcares no néctar, utilizando microsseringas de $5 \mu \mathrm{L}$ e $10 \mu \mathrm{L}$ $\left(\right.$ Microliter $^{\circledR}$ ) e refratômetro de bolso $0-32 \%$ e $28-62 \%$ $\left(\right.$ Atago $^{\circledR}$ ), respectivamente. Para isso, foram previamente ensacados botões $(n=30)$ no dia anterior à antese, de diferentes indivíduos, dos quais dez foram utilizados para a medição de volume e concentração de néctar a cada duas horas durante o primeiro dia de antese (das $05 \mathrm{~h} 00$ às $18 \mathrm{~h} 00$ ), dez permaneceram ensacados sendo as medidas realizadas ao 
final da antese e os outros dez botões foram utilizados para a medição do volume de néctar produzido ainda no estágio de botão. A receptividade da região estigmática foi determinada através da aplicação da solução de permanganato de potássio $\left(\mathrm{KMnO}_{4}, 0,25 \%\right)$ na cabeça do estilete de cinco botões em dia de pré-antese (com anteras deiscentes) de diferentes indivíduos (Robinsohn 1924). A cabeça do estilete foi classificada de acordo com os tipos propostos por Schick (1982) e Fallen (1986).

O número de flores abertas por inflorescência foi quantificado em dez inflorescências jovens de indivíduos diferentes e a duração da antese foi monitorada a partir da marcação de dez botões em fase inicial de abertura, sendo acompanhado o seu ciclo até a senescência floral.

Sistema reprodutivo - Para análise do sistema reprodutivo, foram realizadas em campo polinizações controladas utilizando 30 flores para cada tratamento: 1) polinização natural (controle), 2) autopolinização espontânea, 3) autopolinização manual e 4) polinização cruzada manual. Os botões em pré-antese (um dia antes da abertura) utilizados nos tratamentos 2, 3 e 4 foram ensacados para evitar o contato das flores com os visitantes. Para os tratamentos manuais 3 e 4, os grãos de pólen foram depositados na região receptiva de cada flor com o auxílio de fios de náilon de 0,2 mm, simulando a probóscide dos insetos, uma vez que não foi possível emascular as flores. Para a autopolinização, um único fio de náilon foi utilizado para cada flor, sendo este inserido três vezes consecutivas no tubo. Para a polinização cruzada, três fios de náilons diferentes foram inseridos previamente no tubo de três outras flores de diferentes indivíduos para depois serem inseridos nas flores marcadas para o tratamento, evitando-se assim, a autopolinização ou a geitonogamia. Após efetuado os respectivos tratamentos, as flores foram novamente ensacadas e monitoradas até a formação de frutos. Para análise estatística do número de frutos formados entre os tratamentos foi utilizado o teste $t$ através do programa BioEstat 3.0 (Ayres et al. 2003).

Para a análise do crescimento dos tubos polínicos, foram fixadas em FAA em etanol a 50\%, após 6, 12, 24 e 48 horas da polinização, flores de diferentes indivíduos $(n=15)$ as quais foram separadamente submetidas aos tratamentos manuais de autopolinização e polinização cruzada. Para cada horário de tratamento foi utilizado um conjunto de 10 flores, totalizando 40 flores para cada tratamento. Após a coleta e fixação, os pistilos foram amolecidos e clarificados em solução de hidróxido de sódio $9 \mathrm{~N}$, a $60^{\circ} \mathrm{C}$ por $10 \mathrm{~min}$, lavados, dissecados, corados com azul de nailina e observados sob microscópio de fluorescência (Martin 1959). Foram analisados os seguintes aspectos: germinação dos grãos de pólen na região estigmática, crescimento dos tubos polínicos número de óvulos fecundados para as flores de cada horário e tratamento.

Visitantes florais - O comportamento dos visitantes florais foi registrado em campo através de observações diretas em plantas focais e também da análise de fotografias, totalizando
120 horas de observações diurnas e 30 horas noturnas, distribuídas durante a antese. Através das observações, pode-se determinar o recurso floral procurado, a frequência das visitas durante o dia e o tempo que os insetos permaneciam nas flores. A frequência dos visitantes florais foi estimada através da contagem direta das visitas diurnas (das $05 \mathrm{~h} 00$ às 18h00) às flores de primeiro dia, nas plantas focais. A observação da presença ou ausência de grãos de pólen sobre a língua dos visitates florais foi feita sob estereomicroscópio com os espécimes coletados nos dias das observações de frequência dos visitantes.

Os visitantes foram classificados como: polinizador principal, aquele que conseguia contactar as estruturas reprodutivas, que mostrou-se bastante frequente em suas visitas ( $>10$ visitas horas $\left.{ }^{-1}\right)$ e que apresentava grãos de pólen em sua língua, sendo considerado, portanto, o polinizador mais efetivo; polinizador secundário, aquele que contactava as estruturas reprodutivas, porém menos frequente $(<10$ visitas horas $^{-1}$ ) e que apresentava ou não grãos de pólen em sua língua; ou pilhador, aquele visitante que não contactava as estruturas reprodutivas e roubava o recurso floral. Os insetos foram coletados, montados a seco para identificação e depositados na Coleção Entomológica do Laboratório de Biologia Floral e Reprodutiva, Universidade Federal de Pernambuco, como espécimes-testemunho.

\section{Resultados}

Fenologia reprodutiva - A floração de A. blanchetii é anual e iniciou-se em fevereiro, período que coincide com a estação chuvosa na RPPN, e se estendeu até o fim de julho, com pico nos meses de março e abril (figura 1). A frutificação se seguiu à floração e estendeu-se até o mês de setembro, pois o fruto leva até quarenta dias para amadurecer e, depois, permanece aberto na planta por até pouco mais de um mês aberto dispersando suas sementes pelo vento (anemocoria). Verificou-se que há correlação positiva $\left(\mathrm{r}_{\mathrm{s}}=0,63 ; \mathrm{t}=2,45 ; P=0,03\right)$ entre a precipitação pluviométrica e a floração. $O$ cálculo do índice de sincronia de floração para a população $(Z=0,62)$ indicou que ocorre sincronia média de floração entre os indivíduos da população estudada.

Biologia floral - Allamanda blanchetii é uma espécie arbustiva cujas inflorescências abrem, no máximo, duas flores por dia. As flores são hermafroditas e a corola gamopétala, com cinco pétalas de coloração rosa púrpura, é infundibuliforme, apresentando plataforma de pouso (cada pétala) com uma média de $30 \mathrm{~mm}$.

O tubo da corola é dividido em duas regiões (figura 2), a primeira mais larga abriga o inseto durante a visita e a segunda região, após o cone de anteras, só permite a passagem da língua do visitante floral. $\mathrm{O}$ 


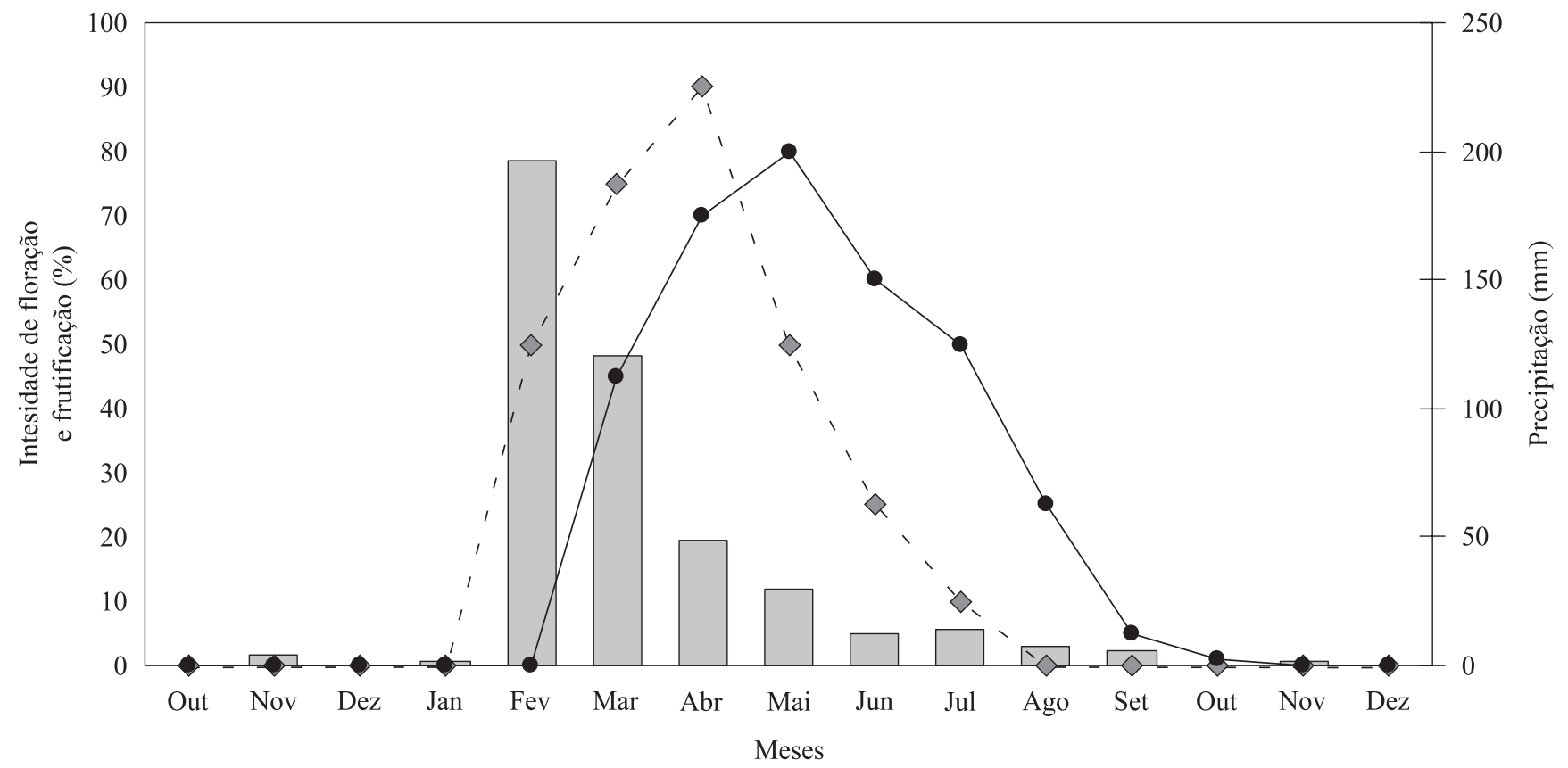

Figura 1. Intensidade de precipitação (mm; $\square)$, floração (\%; - -$)$, frutificação (\%; - -) de Allamanda blanchetii na RPPN Fazenda Almas, Paraíba, Brasil, no período de outubro/2006 a dezembro/2007. Dados climatológicos: Aesa (Agência Executiva de Gestão das Águas do Estado da Paraíba).

Figure 1. Intensity of precipitation (mm; $\square)$, flowering $(\% ;-\diamond-)$, fruiting $(\% ;-\bullet-)$ of Allamanda blanchetii in the RPPN Almas Farm, Paraíba, Brazil, from October/2006 to December/2007. Climatological data: from Aesa (executive agency of water management of the state of Paraíba).

androceu é constituído por cinco estames epipétalos, com anteras formando um cone acima da cabeça do estilete, sobre a qual ainda na fase de botão o pólen é depositado, caracterizando apresentação secundária de pólen (figura 3). A região estigmática (parte receptiva) se localiza na porção inferior da cabeça do estilete. A disposição das anteras, formando o cone, dá origem a cinco aberturas que dão acesso ao néctar (figura 4). A cabeça do estilete, portanto, tem três regiões morfofuncionais: a) a parte superior, estéril, onde o pólen é depositado (apresentação secundária de pólen) e que se encontra coberta pelo cone de anteras; b) a região mediana lateral, onde a epiderme secreta uma substância pegajosa e c) região basal, que constitui o "colarinho" (dilatação das células epidérmicas), abaixo da qual está a região receptiva aos grãos de pólen durante a polinização (estigma funcional) (figura 5).

A flor possui dois ovários (gineceu bicarpelar apocárpico), com uma média total de $50 \pm 2,0$ óvulos. A média do número de grãos de pólen por flor foi de $8.420 \pm 201,3$ com uma viabilidade polínica de $99,4 \%$. A razão do número de grãos de pólen por óvulos resultou em 168,4 grãos óvulo ${ }^{-1}$. O pólen depositado na cabeça do estilete se apresenta como uma massa de grãos unidos por abundante "pollenkitt". A contagem desse pólen apresentado secundariamente revelou que uma média de $6.730 \pm 303,2$ grãos de pólen (cerca de $80 \%$ ) estão disponíveis para ser transportados pelos visitantes florais. As simulações de visitas com fios de náilon permitiram sugerir que, com cinco visitas efetivas, podem ser retirados quase todos os grãos de pólen apresentados secundariamente. Os demais grãos permanecem nas anteras e não são relocados para a cabeça do estilete após a flor ser visitada, como foi constatado em análise posterior das flores em laboratório.

A antese se inicia por volta das cinco horas da manhã e dura de dois a três dias, quando então a flor cai, restando apenas seu ovário. No dia anterior à antese, os botões já possuem suas anteras deiscentes e a região estigmática se encontra receptiva. Além disso, as flores não exalam odor perceptível ao sentido humano. O volume médio de néctar acumulado durante toda a antese é de $36,6 \mu \mathrm{L}$ e concentração de $45 \%$ de solutos. Allamanda blanchetii inicia a produção de néctar ainda no botão em pré-antese (com anteras deiscentes) apresentando volume médio de $21,7 \mu \mathrm{L}$ e, quando a flor abre, esta já possui o volume acumulado, não mais produzindo o recurso que não é reabsorvido, sendo facilmente observado. 

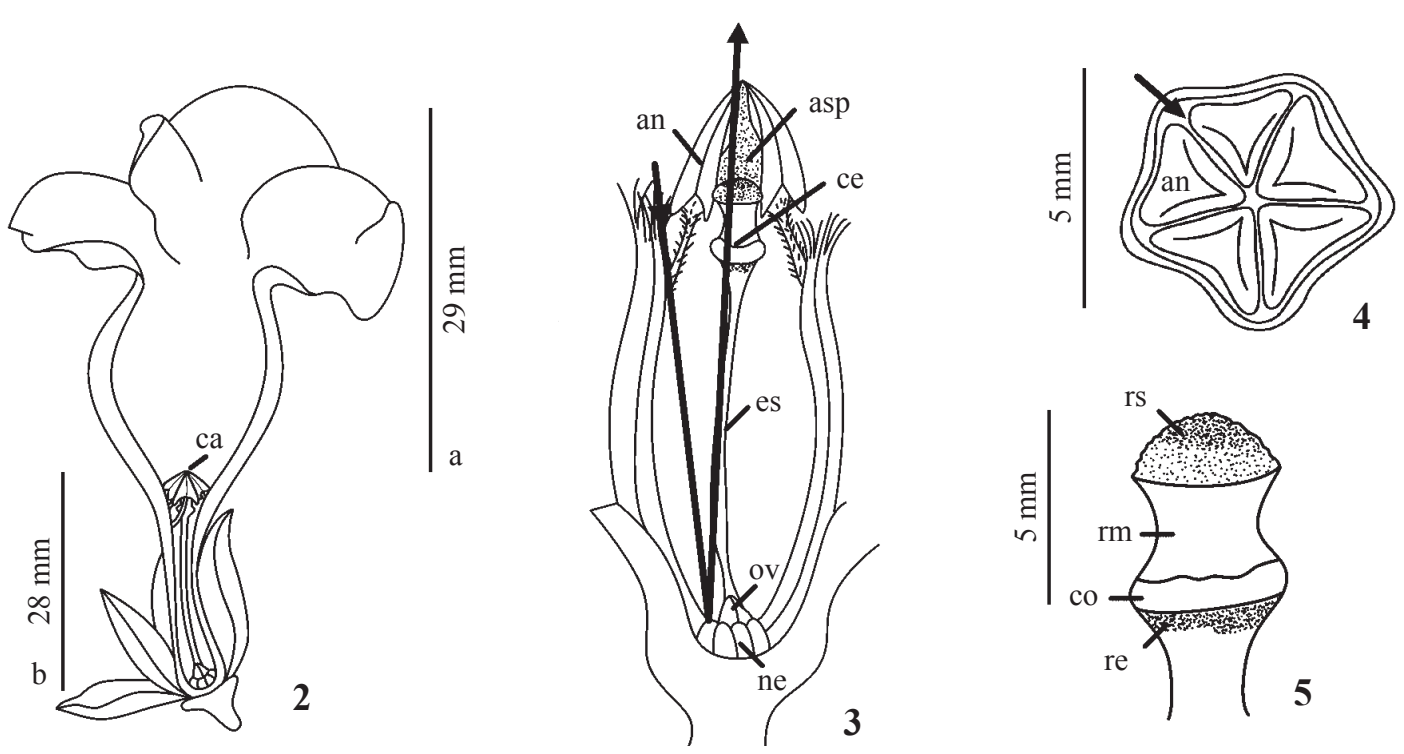

Figuras 2-5. Morfologia floral de Allamanda blanchetii. 2. Corte longitudinal do tubo da corola $(\mathrm{a}=$ região anterior; $\mathrm{b}=$ região posterior; $\mathrm{ca}=$ cone de anteras). 3 . Detalhe do gineceu e androceu (figura 2, parte b); a seta indica a trajetória do inseto ao visitar a flor (an = antera; asp = apresentação secundária de pólen; ce = cabeça do estilete; es = estilete; ov = ovário; ne = nectário). 4. Corte transversal do tubo floral evidenciando o espaço entre as anteras (seta) por onde passa a língua do inseto. 5. Cabeça do estilete ( $\mathrm{rs}=$ região superior estéril; $\mathrm{rm}=$ região mediana; $\mathrm{co}=$ anel basal; re = região inferior e receptiva com pólen).

Figures 2-5. Floral morphology of Allamanda blanchetii. 2. Longitudinal section of the corolla tube $(\mathrm{a}=$ upper region; $\mathrm{b}=$ lower region; $\mathrm{ca}=$ cone of the anthers). 3 . Detail of the gynoecium and androecium (figure 2, part b); the arrow indicates the trajectory of the insect tongue when visits the flower ( $\mathrm{an}=$ anthers; asp = secondary pollen presentation; ce = style head; es = style; ov $=$ ovary; ne = nectary). 4 . Cross section of the floral tube showing the space among the anthers (arrow) for the passage of the tongue of the visiting insect. 5 . Style head $(\mathrm{rs}=$ apical sterile region; $\mathrm{rm}=$ middle region; $\mathrm{co}=$ basal ring; $\mathrm{re}=$ basal and receptive region with pollen).

Sistema reprodutivo - Os resultados dos experimentos de polinização (tabela 1 ) revelaram que $A$. blanchetii é auto-incompatível, uma vez que não houve a formação de frutos nos experimentos de autopolinização manual. Não houve diferença significativa entre os tratamentos

Tabela 1. Polinização, frutificação e produção de sementes sob diferentes tratamentos em Allamanda blanchetii na RPPN Fazenda Almas, São José dos Cordeiros, Paraíba, Brasil.

Table 1. Pollination, fruit and seed set in Allamanda blanchetii under different treatments at the RPPN Fazenda Almas, São José dos Cordeiros, Paraíba State, Brazil.

\begin{tabular}{lcc}
\hline Tratamentos & $\begin{array}{c}\text { Flores/Frutos/ } \\
\text { Sementes } \\
(n)\end{array}$ & $\begin{array}{c}\text { Sucesso } \\
(\%)\end{array}$ \\
\hline Condições naturais & $30 / 10 / 367$ & 33,3 \\
Autopolinização espontânea & $30 / 0 / 0$ & 0 \\
Autopolinização manual & $30 / 0 / 0$ & 0 \\
Polinização cruzada manual & $30 / 12 / 422$ & 40,0 \\
\hline
\end{tabular}

de polinização cruzada e sob condições naturais $(\mathrm{t}=1,1$, $\mathrm{g}=5, P=0,5)$. Nos experimentos de autopolinização manual, observou-se que os ovários murchavam e caíam no estágio inicial do desenvolvimento do fruto.

$\mathrm{Na}$ análise de crescimento dos tubos polínicos, foi verificado que seis horas após a polinização, já podem ser vistos grãos de pólen germinando na região estigmática (figura 6) e após doze horas os tubos já haviam penetrado nos óvulos (figura 7) em ambos os tratamentos. No entanto, o número de óvulos penetrados no tratamento de polinização cruzada foi proporcionalmente maior, havendo diferença significativa na frequência de óvulos penetrados $\left(\chi^{2}=17,988\right.$, g.l. $\left.=1, P<0,0001\right)$ (tabela 2$)$. $\mathrm{O}$ fruto é uma cápsula bivalvar, desenvolvido de dois carpelos, cujas sementes são dispersas pelo vento. A partir da análise dos frutos oriundos dos testes de polinização, observou-se que em condições naturais os frutos têm em média $36 \pm 1,2$ sementes formadas.

Visitantes florais - As flores de A. blanchetii foram visitadas por três espécies de abelhas, quatro de borboletas e duas de beija-flores (tabela 3). As abelhas iniciavam 

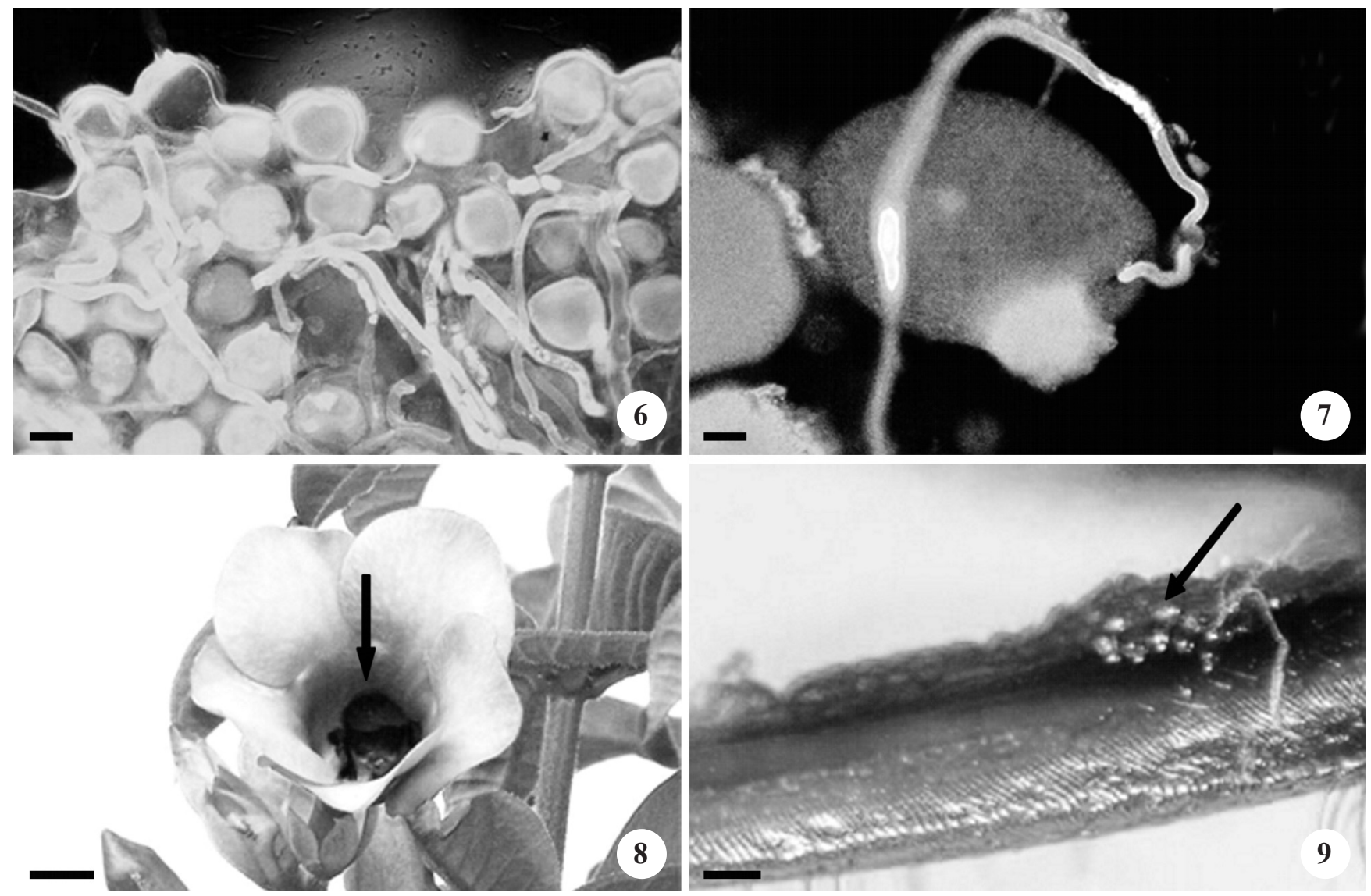

Figuras 6-9. Allamanda blanchetii. 6. Grãos de pólen do tratamento de autopolinização manual germinando na região estigmática 6 horas após a polinização. 7. Óvulo com tubo polínico oriundo de autopolinização manual 12 horas após a polinização. 8. Eulaema nigrita (seta) visitando uma flor. 9. Grãos de pólen (seta) de A. blanchetii sobre a glossa de E. nigrita. Barra $=100 \mu \mathrm{m}$ (6-7), 1,0 cm (8), 0,5 mm (9).

Figures 6-9. Allamanda blanchetii. 6. Pollen grains of manual self-pollination treatment germinating in the stigmatic region six hours after pollination. 7. Ovule with pollen tube from manual self-pollination 12 hours after pollination. 8. Eulaema nigrita (arrow) visiting the flower. 9. Pollen grains (arrow) of A. blanchetii on the glossa of E. nigrita. Bar $=100 \mu \mathrm{m}(6-7), 1.0 \mathrm{~cm}$ (8), $0.5 \mathrm{~mm}(9)$.

as visitas entre $5 \mathrm{~h} 00$ e $6 \mathrm{~h} 00$ da manhã, forrageando até o fim da tarde, enquanto as borboletas iniciavam suas visitas entre $7 \mathrm{~h} 00$ e $8 \mathrm{~h} 00$ da manhã, intensificando-as entre $9 \mathrm{~h} 00$ e $11 \mathrm{~h} 30$ e, mais esporadicamente, até o fim da tarde.

Entre as espécies de abelha, Eulaema nigrita (Euglossini) (figura 8) foi considerada o principal polinizador de $A$. blanchetii, uma vez que esta foi mais frequente em suas visitas (figura 10). Além disso, em todos os indivíduos coletados havia grãos de pólen de $A$. blanchetii em sua glossa, na porção proximal (figura 9). Durante as observações foi percebido que E. nigrita parecia apresentar rotas de forrageamento, em um comportamento conhecido na literatura como trapliners. Ao avistar as flores, E. nigrita pairava frontalmente por alguns segundos e entrava no tubo floral, permanecendo por até 35 segundos em flores jovens (de primeiro dia), quando então se deslocava à procura de outras flores próximas e, em seguida, deixava o indivíduo.

Uma espécie de abelha do gênero Melitoma foi observada visitando as flores de $A$. blanchetii, mas, muito provavelmente, essa abelha não alcançava o néctar devido ao curto cumprimento de sua língua $(11,2 \mathrm{~mm}) \mathrm{em}$ relação à segunda região do tubo floral $(27,7 \mathrm{~mm})$. O seu comportamento a classifica como pilhadora, já que suas várias tentativas de tomada de néctar levaram à perda do pólen, visto que $A$. blanchetii é auto-incompatível. Além disso, a abelha limpava o pólen de sua língua com suas patas dianteiras sempre que finalizava uma tentativa de tomada de néctar. 
Tabela 2. Número de pistilos examinados, óvulos observados e óvulos penetrados $6,12,24$ e 48 horas após experimentos de autopolinização manual e polinização cruzada em flores de Allamanda blanchetii.

Table 2. Number of examined pistils, observed and penetrated ovules $6,12,24$ and 48 hours after manual self- and crosspollination experiments in flowers of Allamanda blanchetii.

\begin{tabular}{cccc}
\hline Experimentos & $\begin{array}{c}\text { Pistilos } \\
\text { examinados }\end{array}$ & $\begin{array}{c}\text { Óvulos } \\
\text { observados }\end{array}$ & $\begin{array}{c}\text { Óvulos } \\
\text { penetrados }\end{array}$ \\
\hline $\begin{array}{c}\text { Autopolinização } \\
\text { manual (h) }\end{array}$ & & & \\
6 & 8 & 284 & 0 \\
$12^{*}$ & 9 & 346 & 126 \\
24 & 8 & 320 & 144 \\
48 & 8 & 344 & 112 \\
Total & 33 & 1294 & 382 \\
Polinização & & & \\
cruzada (h) & & & \\
6 & 8 & 308 & 0 \\
$12^{*}$ & 8 & 220 & 120 \\
24 & 8 & 140 & 72 \\
48 & 8 & 288 & 120 \\
Total & 32 & 956 & 312 \\
\hline
\end{tabular}

${ }^{*} \chi^{2}=17,988, P<0,0001 / * \chi^{2}=17.988, P<0.0001$.

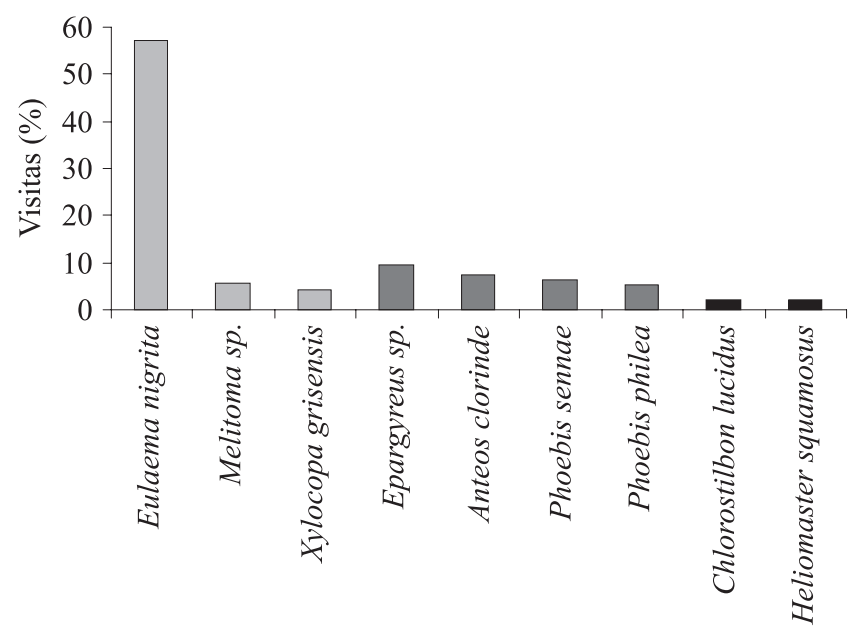

Visitantes

Figura 10. Frequência de visitas realizadas às flores de Allamanda blanchetii na RPPN Fazenda Almas, São José dos Cordeiros/PB.

Figure 10. Frequency of visits to flowers of Allamanda blanchetii at the RPPN Fazenda Almas, São José dos Cordeiros/PB.
Xylocopa grisescens também se comportou como um pilhador, uma vez que foi observada fazendo fissuras laterais externamente ao tubo da corola a procura de néctar. Algumas raras vezes, esta abelha foi vista entrando na flor e, provavelmente, devido a sua glossa curta, não deve ter alcançado o recurso na base do tubo floral, o que justifica as suas raras entradas no tubo da flor e seu comportamento pilhador.

As borboletas foram consideradas polinizadores secundárias, uma vez que foram visitantes menos frequentes e que contactavam as estruturas reprodutivas da flor. Entretanto, nem todos os indivíduos coletados apresentavam grãos de pólen em sua probóscide. As borboletas visitavam até duas flores por indivíduo, quando então voavam para um outro bem mais distante.

Entre as borboletas, a espécie Epargyreus sp. foi a mais frequente e a que apresentou maior quantidade de pólen na sua probóscide (30 $\mathrm{mm}$ de comprimento). As borboletas pousavam na flor e introduziam sua probóscide em uma das cinco entradas existentes entre as anteras, depois penetravam o máximo que

Tabela 3. Visitantes florais de Allamanda blanchetii na RPPN Fazenda Almas, São José dos Cordeiros, Paraíba, Brasil. $(\mathrm{PP}=$ principal polinizador; $\mathrm{PS}=$ polinizador secundário; $\mathrm{PI}=$ pilhador $)$.

Table 3. Floral visitors of Allamanda blanchetii in the RPPN Fazenda Almas, São José dos Cordeiros, Paraíba State, Brazil. $(\mathrm{PP}=$ main pollinator; $\mathrm{PS}=$ secondary pollinator; $\mathrm{PI}=$ nectar thief).

Visitantes Florais

Tipo de visitante

Himenópteros

Apidae

Eulaema nigrita Lepeletier, 1841

PP

Anthophoridae

Xylocopa grisescens Lepeletier, 1841

Melitoma sp.

PI

PI

Lepidópteros

Pieridae

Anteos clorinde Godart, 1824

PS

Phoebis sennae Linnaeus, 1758

PS

Phoebis philea Linnaeus, 1763

PS

Hesperiidae

Epargyreus sp.

PS

Aves

Trochilidae

Chlorostilbon lucidus Shaw, $1812 \quad$ PI

Heliomaster squamosus Temminck, 1823 
suas asas permitiam no tubo da corola. Epargyreus sp. permanecia por até um minuto nas flores jovens (de primeiro dia) e, após uma visita, procurava outras flores próximas.

Os beija-flores Chlorostilbon lucidus e Heliomaster squamosus foram observados pilhando secundariamente néctar das flores de $A$. blanchetii, uma vez que se aproveitavam das fissuras externas feitas pela abelha $X$. grisescens. Os beija-flores também foram observados inserindo a cabeça na primeira região do tubo floral, permanecendo geralmente cerca de dois segundos na flor. Provavelmente, os beija-flores pareciam atraídos pela flor e poderiam estar tentando acessar o recurso floral ou à procura de pequenos insetos.

No período noturno, observou-se que as flores de $A$. blanchetii foram visitadas por esfingídeos, os quais não puderam ser coletados e, consequentemente, identificados, em decorrência de suas raras e rápidas visitas. Estes podem ter eventualmente contatado as partes reprodutivas quando tentavam tomar néctar e suas visitas aconteceram, geralmente, no fim da noite e, mais comumente, em dias de lua cheia.

\section{Discussão}

Fenologia reprodutiva - As fenofases de floração (fevereiro a julho) e de frutificação (fevereiro a setembro) de Allamanda blanchetii apresentaram padrão do tipo anual (sensu Newstrom et al. 1994) e do tipo 2 ou steady state segundo Gentry (1974), no qual há uma produção constante de poucas flores por dia durante um extenso período. Esse padrão de floração sugere que $A$. blanchetii seja uma importante fonte de recurso alimentar para seus polinizadores, principalmente, em ambientes com condições extremas como a Caatinga, onde pode ocorrer a escassez de recursos por longos períodos (Machado \& Lopes 2004, Machado \& Sazima 2008). O padrão de floração steady state está relacionado à plantas que são visitadas por polinizadores com rotas fixas de forrageamento e que cobrem longas distâncias, chamados trapliners (Gentry 1974, Endress 1994). Polinizadores trapliners são importantes para A. blanchetii, uma vez que esta espécie apresenta propagação vegetativa e, portanto, tal comportamento reduz a geitonogamia e, consequentemente, também reduz a perda de pólen, uma vez que a espécie é autoincompatível.

A forte correlação positiva entre a floração de $A$. blanchetii e a precipitação demostra como a água é um fator importante não só por ativar a floração como também por mantê-la. A sazonalidade é um evento marcante na fenologia reprodutiva das espécies da Caatinga, influenciada principalmente pela precipitação (Griz \& Machado 2001, Barbosa et al. 2003). O fato do período de frutificação se estender um pouco mais do que o da floração, coincidindo com o início da estação seca, bem como o fato do fruto levar muito tempo para se desenvolver e entrar em estágio de maturação, resulta na estratégia reprodutiva de dispersar suas sementes anemocóricas na época seca.

Os indivíduos da população de $A$. blanchetii apresentaram sincronia média de floração de acordo com a classificação de Augspurger (1983). Segundo este autor, tal amplitude de sincronia ocorre em espécies que apresentam o padrão de floração steady state (sensu Gentry 1974). Essa sincronia de amplitude média entre os indivíduos favorece e aumenta o "display visual", podendo atrair mais polinizadores.

Biologia floral - As flores de A. blanchetii apresentam características florais relacionadas às síndromes de polinização por abelhas (melitofilia), tais como antese diurna e elevada concentração de néctar, e por borboletas (psicofilia), como corola tubulosa de cor rosa claro a rosa púrpura com plataforma de pouso e antese diurna (Faegri \& van der Pij1 1979, Endress 1994, Proctor et al. 1996). A amplitude de atributos florais apresentados por A. blanchetii parece favorecer a visita por esses dois grupos de insetos.

Morfologicamente, a flor de $A$. blanchetii é bastante especializada devido à sua hercogamia e à apresentação secundária de pólen. Além disso, a flor é dividida em duas regiões que "selecionam" os visitantes florais por tamanhos compatíveis com o diâmetro da primeira região e com o comprimento do tubo da corola da segunda região. Segundo Barrows (1976) e Schemske (1976), o comprimento do tubo da corola e a localização do néctar na base do tubo podem determinar o tipo de visitante capaz de explorar esse recurso.

$\mathrm{O}$ arranjo espacial das estruturas reprodutivas de $A$. blanchetii caracteriza sua forte hercogamia que, segundo Schick (1982), direciona o aparelho bucal do polinizador, evitando a autopolinização. Entretanto, foi verificado em outros estudos com outras espécies de Apocynaceae (Löhne et al. 2004) que esta hercogamia nem sempre evita a autopolinização mediada por insetos, caso estes insiram seu aparelho bucal várias vezes na mesma flor visitada. No caso de A. blanchetii essa autopolinização mediada por insetos também pode ocorrer, como foi descrito no comportamento da abelha do gênero Melitoma, e embora esse comportamento leve a uma perda de 
pólen, devido a auto-incompatibilidade da espécie, isso não afeta, significativamente, o seu sucesso reprodutivo.

$\mathrm{Na}$ apresentação secundária de pólen de $A$. blanchetii, este encontra-se "escondido" no cone de anteras e sobre a cabeça do estilete, numa quantidade de aproximadamente $80 \%$ do total de pólen das anteras, significando que nem todo o pólen permanece disponível e, portanto, a coleta e o transporte devem ser realizados de forma a maximizar a sua deposição na parte receptiva da flor, justificando, provavelmente, a grande quantidade de pollenkitt formado pela flor. Segundo Yeo (1993), a localização e a não exposição dos grãos de pólen nas Apocynaceae s.str. evitam o seu roubo e permitem que estes sejam depositados corretamente nas partes bucais do polinizador. A morfologia da cabeça do estilete de $A$. blanchetii tem as três regiões bem distintas, caracterizando o tipo "Allamanda" proposto por Schick (1982) e o tipo "Nível 2" descrito por Fallen (1986), no qual durante sua retração, o aparelho bucal do inseto raspa no anel basal ("colarinho") da cabeça do estilete, peculiar nesse grupo de plantas.

A baixa razão $\mathrm{P} / \mathrm{O}$ apresentada pela espécie permitiu classificá-la como autógama facultativa (Cruden 1977). Porém, ela se comporta como uma espécie xenogâmica, uma vez que necessita obrigatoriamente de vetores bióticos para a sua polinização em decorrência, principalmente, da sua auto-incompatibilidade. As espécies com apresentação secundária de pólen tratadas por Cruden (1977) também apresentaram razão P/O abaixo da média obtida para as espécies xenogâmicas. O eficiente mecanismo de polinização, o qual minimiza a perda de pólen através de mecanismos auxiliares de aderência de pólen ao visitante, deve ser responsável pela baixa razão $\mathrm{P} / \mathrm{O}$ de muitas espécies xenogâmicas na família (Yeo 1993).

A concentração de açúcares no néctar $(45 \%)$ de A. blanchetii é compatível com os valores relatados para flores polinizadas por abelhas (40-49\%), mas está fora da faixa de variação para flores polinizadas por borboletas que tendem a oferecer néctar mais diluído (15-25\%) (Proctor et al. 1996). A produção de néctar nos botões em estágio de abertura pode ser justificada pela coincidência de horário entre o início da antese e o início das visitas das abelhas, ou seja, entre cinco e seis horas da manhã, ao abrirem, as flores já dispõem o recurso para as abelhas aumentando, assim, o número de visitas nessas flores. Provavelmente, o fato das abelhas iniciarem o forrageamento antes das borboletas tenha sido a razão pelo pouco pólen encontrado nas probóscides das borboletas coletadas.
Sistema reprodutivo - Os experimentos revelaram que A. blanchetii necessita de vetores bióticos para a sua polinização. $\mathrm{O}$ fato de não haver diferença significativa entre os resultados dos tratamentos de controle e de polinização cruzada manual sugere que os visitantes florais sejam frequentes em suas visitas e eficientes na remoção, no transporte e na deposição do pólen na parte receptiva da cabeça do estilete, caracterizando-se, portanto, como polinizadores efetivos de $A$. blanchetii.

O número de óvulos penetrados, o crescimento de tubos polínicos até o óvulo das flores submetidas aos tratamentos de autopolinização manual e a queda dos ovários em estágio inicial do desenvolvimento, observado nos testes manuais de autopolinização, sugerem que $A$. blanchetii possa apresentar o mecanismo de auto-incompatibilidade de ação tardia (sensu Seavey \& Bawa 1986, Waser \& Price 1991). Este mecanismo já foi registrado para outras espécies da família Apocynaceae tais como Aspidosperma macrocarpon Mart. (Gibbs 1990), Rauvolfia grandiflora Mart. (Lopes \& Machado 1999), Apocynum cannabinum L. (Lipow \& Wyatt 1999) e várias espécies do gênero Asclepias (Kahn \& Morse 1991, Sage \& Williams 1991, Lipow \& Wyatt 1998).

$\mathrm{Na}$ análise do crescimento dos tubos polínicos das flores submetidas ao tratamento de autopolinização manual observam-se muitos grãos de pólen germinando na região estigmática, fato que pode interferir no crescimento dos tubos polínicos oriundos de polinização cruzada, causando uma oclusão de área receptiva (Lloyd \& Yates 1982). Assim, mecanismos que previnam ou minimizem a autopolinização nas espécies autoincompatíveis parecem ser vantajosos (Lopes \& Machado 1999), no caso de $A$. blanchetii, a sua forte hercogamia.

Visitantes florais - Com base na alta frequência, no comportamento das visitas, nas análises de presença de grãos de pólen na língua e na morfologia floral, podemos inferir que a abelha Eulaema nigrita foi o principal polinizador de A. blanchetii. Segundo Schlindwein et al. (2004), por possuírem glossa longa, as abelhas Euglossini são capazes de explorar flores tubulares não acessíveis a outras abelhas. O comportamento trapliner aparentemente apresentado por E. nigrita favorece a polinização cruzada, sendo isto um fator muito importante pois a espécie apresenta propagação vegetativa e é auto-incompatível. O comportamento trapliner é de grande importância por favorecer a polinização cruzada entre plantas tropicais (Janzen 1971) e é um comportamento muito comum nas abelhas 
pertencentes a tribo Euglossini (Waddington 1983). De acordo com Janzen (1971), a família Apocynaceae constitui uma importante fonte de néctar para as abelhas desta tribo.

O comportamento pilhador da abelha Xylocopa grisescens está associado ao tamanho da segunda região do tubo floral e ao reduzido tamanho da sua língua. De acordo com Vieira et al. (1992), as abelhas do gênero Xylocopa possuem glossa curta e por isto não conseguem alcançar o recurso na base de flores tubulares. Além disso, espécies tropicais de Xylocopa são comumente relatadas como pilhadoras de néctar de flores tubulares (Gobatto-Rodrigues \& Stort 1992, Lopes et al. 2002).

O mecanismo de polinização de $A$. blanchetii é semelhante ao descrito por Schick (1982) e Fallen (1986) para outras Apocynaceae, no qual o polinizador insere sua língua em uma das cinco entradas formadas pelo cone de anteras, sendo "guiado" durante a retração da língua a raspar no anel basal da cabeça do estilete ("colarinho") depositando o pólen exógeno na região estigmática. Ainda durante a retração, a língua passa na região mediana da cabeça do estilete, onde entra em contato com a substância pegajosa e, por fim, passa pelo centro do cone de anteras, onde coleta novo conjunto de pólen para uma próxima visita (ver seta da figura 3).

As borboletas foram consideradas polinizadores secundários, pois mesmo contactando a região estigmática da flor, estas não mostram uma frequência de visitas tão alta quando comparada com a frequência de E. nigrita. No entanto, o comportamento de visitar as flores de um indivíduo e voar para outro mais distante é importante por manter o fluxo gênico entre as subpopulações de A. blanchetii. Borboletas já foram relatadas como polinizadores que cobrem longas distâncias com rotas fixas de forrageamento (Waddington 1976, Cruden \& Hermann-Parker 1979).

Embora reduza a quantidade de néctar para os polinizadores, a pilhagem por Xylocopa grisescens e pelos beija-flores não reduz a atração visual das flores de $A$. blanchetii, sendo estas, portanto, visitadas pelos polinizadores mesmo quando já não há mais nenhum recurso disponível. Neste caso, os polinizadores, presumivelmente, são obrigados a visitar mais flores à procura de néctar, aumentando as chances de polinização cruzada. Essa pilhagem, portanto, pode ter um efeito positivo sobre o sucesso reprodutivo de $A$. blanchetii uma vez que a flor não mais produz néctar após este ser retirado. Sazima (1981) também observou que a presença dos pilhadores de Pavonia montana Garcke
(Malvaceae) não reduziram a atração de suas flores em relação aos polinizadores, resultando também no aumento das visitas e, consequentemente, no aumento da polinização cruzada.

As raras visitas de esfingídeos devem ser devidas ao fato das flores de $A$. blanchetii não possuírem atributos que atraiam lepidópteros noturnos como odores fortes e cores claras, além disso, a disponibilidade de néctar depende das visitas que a flor recebe durante o dia.

O padrão de floração e a morfologia floral, influenciando o comportamento dos visitantes florais, refletem o especializado mecanismo de polinização de Allamanda blanchetii. Além disso, a alta frequência e eficiência dos polinizadores primário e secundário podem compensar os efeitos negativos da geitonogamia em função da auto-incompatibilidade, sendo responsáveis e indispensáveis para garantir o sucesso reprodutivo da espécie.

Agradecimentos - Ao Dr. André Victor L. Freitas (Unicamp), pela identificação das espécies de borboletas; ao PELD/CNPq, pelo apoio para o desenvolvimento da pesquisa de campo; à Capes, pela bolsa de Mestrado concedida à primeira autora, e ao Dr. Marcelo Guerra, pela permissão dada para o uso do microscópio de fluorescência no Laboratório de Citogenética (UFPE).

\section{Referências bibliográficas}

AGUIAR, J., LACHER, T. \& SILVA, J.M.C. 2002. The Caatinga. In Wilderness - earth's last wild places (P.R. Gil, ed.). Cemex, Cidade do México, p.174-181.

ALBERS, P. \& VAN DER MAESEN, L.J.G. 1994. Pollination of Apocynaceae. Wageningen Agricultural University Papers 94:61-81.

AUGSPURGER, C.K. 1983. Phenology, flowering synchrony, and fruit set of six Neotropical shrubs. Biotropica 15:257-267.

AYRES, M., AYRES JÚNIOR, M., AYRES, D.L. \& SANTOS, A.S. 2003. BioEstat 3.0. Aplicações estatísticas nas áreas das ciências biológicas e médicas. Sociedade Civil Mamirauá/ MCT - CNPq/ Conservation International, Belém.

BARBOSA, D.C.A., BARBOSA, M.C.A. \& LIMA, L.C.M. 2003. Fenologia de espécies lenhosas da Caatinga. In Ecologia e conservação da Caatinga (I.R. Leal, M. Tabarelli \& J.C.M. Silva, eds.). Universidade Federal de Pernambuco, Recife, p.657-693.

BARROWS, E.M. 1976. Nectar robbing and pollination of Lantana camara (Verbenaceae). Biotropica 8:132-135.

CRUDEN, R.W. 1977. Pollen-ovule rations: a conservative indicator of breeding systems in flowering plants. Evolution 31:32-46. 
CRUDEN, R.W. \& HERMANN-PARKER, S.M. 1979. Butterfly pollination of Caesalpinia pulcherrima, with observations on a psychophilous syndrome. Journal of Ecology 67:155-168.

DARRAULT, R.O. \& SCHLINDWEIN, C. 2005. Limited fruit production in Hancornia speciosa (Apocynaceae) and pollination by nocturnal and diurnal insects. Biotropica 37:381-388.

ENDRESS, P.K. 1994. Diversity and evolutionary biology of tropical flowers. Cambridge University Press, Cambridge.

ENDRESS, M.E. \& BRUYNS, P.V. 2000. A revised classification of the Apocynaceae s.l. The Botanical Review 66:1-56.

FAEGRI, K. \& VAN DER PIJL, L. 1979. The principles of pollination ecology. Pergamon Press, London.

FALLEN, M.E. 1985. The gynoecial development and systematic position of Allamanda (Apocynaceae). American Journal of Botany 72:572-579.

FALLEN, M.E. 1986. Floral structure in Apocynaceae: morphological, functional and evolutionary aspects. Botanischer Jahrbucher Systematik 106:245-286.

FOURNIER, L.A. 1974. Un método cuantitativo para la medición de características fenologicas en árboles. Turrialba 24:422-423.

GENTRY, A.H. 1974. Flowering phenology and diversity in tropical Bignoniaceae. Biotropica 6:64-68.

GIBBS, P. 1990. Self-incompatibility in flowering plants: a neotropical perspective. Revista Brasileira de Botânica 13:125-136.

GIULIETTI, A.M., HARLEY, R.M., QUEIROZ, L.P., BARBOSA, M.R.V., BOCAGE NETA, A.L. \& FIGUEIREDO, M.A. 2002. Espécies endêmicas da caatinga. In Vegetação \& flora da Caatinga (E.V.S.B. Sampaio, A.M. Giulietti, J. Virginio \& C.F.L. GamarraRojas, eds.). Associação Plantas do Nordeste - APNE/ Centro Nordestino de Informações sobre Plantas - CNIP/ Universidade Federal de Pernambuco, Recife, p.103-115.

GOBATTO-RODRIGUES, A.A. \& STORT, M.N.S. 1992. Biologia floral e reprodução de Pyrostegia venusta (Ker-Gawl.) Miers (Bignoniaceae). Revista Brasileira de Botânica 15:37-41.

GRIZ, L.M.S. \& MACHADO, I.C. 2001. Fruiting phenology and seed dispersal syndromes in caatinga, a tropical dry forest in the northeast of Brazil. Journal of Tropical Ecology 17:303-321.

HABER, W.A. 1984. Pollination by deceit in a mass-flowering tree Plumeria rubra L. (Apocynaceae). Biotropica 16:269-275.

HERRERA, J. 1991. The reproductive biology of a riparian Mediterranean shrub Nerium oleander L. (Apocynaceae). Botanical Journal of Linnean Society 106:147-172.

HOWELL, G.J., SILATER, A.T. \& KNOX, R.B. 1993. Secondary pollen presentation in Angiosperms and its biological significance. Australian Journal of Botany 41:417-438.
JANZEN, D.H. 1971. Euglossini bees as long-distance pollinators of tropical plants. Science 171:203-205.

KAHN, A.P. \& MORSE, D.H. 1991. Pollinium germination and putative ovule penetration in self- and crosspollinated common milkweed, Asclepias syriaca. American Midland Naturalist 126:61-67.

KOCH, I., BITTRICH, V. \& KINOSHITA, L.S. 2002. Reproductive biology and functional aspects of the floral morphology of Rauvolfia sellowii Müll. Arg. (Apocynaceae; Ruvolfioideae) - a report of dioecy in Apocynaceae. Botanische Jahrbücher 124:83-104.

LIN, S. \& BERNARDELLO, G. 1999. Flower structure and reproductive biology in Aspidosperma quebrachoblanco (Apocynaceae), a tree pollinated by deceit. International Journal of Plant Sciences 160:869-878.

LINHART, Y.B. \& FEINSINGER, P. 1980. Planthummingbird interactions: effects of island size and degree of specialization on pollination. Journal of Ecology 68:745-760.

LIPOW, S.R. \& WYATT, R. 1998. Reproductive biology and breeding system of Gonolobus suberosus (Asclepiadaceae). Journal of the Torrey Botanical Society 125:183-193.

LIPOW, S.R. \& WYATT, R. 1999. Floral morphology and late-acting self-incompatibility in Apocynum cannabinum (Apocynaceae). Plant Systematics and Evolution 219:99-109.

LLOYD, D.G. \& YATES, C.J. 1982. Intrasexual selection and segregation of pollen and stigmas in hermaphrodite plants, exemplified in Wahlenbergia albomarginata (Campanulaceae). Evolution 36:909-913.

LÖHNE, C., MACHADO, I.C.S., POREMBSKI, S., ERBAR, C. \& LEINS, P. 2004. Pollination biology of a Mandevilla species (Apocynaceae), characteristic of NE - Brazilian inselberg vegetation. Botanischer Jahrbucher Systematik 125:229-243.

LOPES, A.V. \& MACHADO, I.C. 1999. Pollination and reproductive biology of Rauvolfia grandiflora (Apocynaceae) secondary pollen presentation, herkogamia and self-incompatibility. Plant Biology 1:547-553.

LOPES, A.V., VOGEL, S. \& MACHADO, I.C. 2002. Secretory trichomes a substitutive floral néctar source in Lundia A. DC. (Bignoniaceae) a genus lacking a functional disc. Annals of Botany 90:169-174.

MACHADO, I.C. \& LOPES, A.V. 2004. Floral traits and pollination systems in the Caatinga, a Brazilian tropical dry forest. Annals of Botany 94:365-376.

MACHADO, I.C.S. \& SAZIMA, M. 2008. Pollination and breeding system of Melochia tomentosa L. (Malvaceae), a keystone floral resource in the Brazilian Caatinga. Flora 203:484-490.

MARTIN, F.W. 1959. Staining and observing pollen tubes in the style by means of fluorescence. Stain Technology $34: 125-128$. 
MMA - Ministério do Meio Ambiente. 2002. Avaliação e ações prioritárias para a conservação da biodiversidade da Caatinga. Universidade Federal de Pernambuco/ Fundação de Apoio ao Desenvolvimento/Conservation International do Brasil, Fundação Biodiversitas, EMBRAPA/Semi-Árido, MMA/SBF, Brasília.

NEWSTROM, L.E., FRANKIE, G.W. \& BAKER, H.G. 1994. A new classification for plant phenology based on flowering patterns in lowland Tropical Rain Forest Trees at La Selva, Costa Rica. Biotropica 26: 141-159.

PROCTOR, M., YEO, P. \& LACK, A. 1996. The natural history of pollination. Timber Press, Portland, Oregon.

RADFORD, A.E., DICKINSON, W.C., MASSEY, J.R. \& BELL, C.R. 1974. Vascular plant systematics. Harper \& Row Publishers, New York.

RAJU, A.J.S., ZAFAR, R. \& RAO, S.P. 2005. Floral device for obligate selfing by remote activity and anemochory in Wrightia tinctoria (Roxb.) R. Br. (Apocynaceae). Current Science 88:1378-1380.

ROBINSOHN, I. 1924. Die Färbungsreaktion der Narbe, Stigmatochromie, als Morpho-biologische Blütenuntersuchungsmethode. Sitzungsberichten Akadenuscher Wissduschaft Wein. Mathematics 133:180-213.

SAGE, T.L. \& WILLIAMS E.G. 1991. Self-incompatibility in Asclepias. Plant Cell Incompatibility Newsletter 23:55-57.

SAKANE, M. \& SHEPHERD, G.J. 1986. Revisão do gênero Allamanda L. (Apocynaceae). Revista Brasileira de Botânica 9:125-149.

SAZIMA, M. 1981. Polinização de duas espécies de Pavonia (Malvaceae) por beija-flores, na Serra do Cipó, Minas Gerais. Revista Brasileira de Biologia 41:733-737.

SEAVEY, S.R. \& BAWA, K.J. 1986. Late-acting selfincompatibility in angiosperms. The Botanical Review 52:195-219.

SCHEMSKE, D.W. 1976. Pollinator specificity in Lantana camara and L. trifolia (Verbenaceae). Biotropica 8: 260-264.

SCHICK, B. 1980. Untersuchungen über die Biotechnik der Apocynaceenblüte I. Morphologie und Funktion des Narbenkopfes. Flora 170:394-432.

SCHICK, B. 1982. Untersuchungen über die Biotechnik der Apocynaceenblüte. II. Bau und Funktion des Bestaübungsapparates. Flora 172:347-371.
SCHLINDWEIN, C., DARRAULT, R.O. \& GRISI, T. 2004. Reproductive strategies in two sphingophilous apocynaceous trees attracting pollinators through nectar or deceit. In Results of worldwide ecological studies. Proceedings of the $2^{\text {nd }}$ Symposium of the A.F.W. SchimperFoundation. (S.W. Breckle, B. Schweizer \& A. Fangmeier, eds.). Verlag Günter Heimbach, Stuttgart, p.215-227.

TORRES, C. \& GALETTO, L. 1998. Patterns and implications of floral nectar secretion, chemical composition, removal effects and standing crop in Mandevilla pentlandiana (Apocynaceae). Botanical Journal of the Linnean Society 127:207-233.

TOSTES, R.B., VIEIRA, M.F. \& CAMPOS, L.A.O. 2003. Polinização de Peltastes peltatus (Vell.) Woodson (Apocynoideae, Apocynaceae) por abelhas euglossíneas. In Apoidea Neotropica: homenagem aos 90 anos de Jesus Santiago Moure (G.A.R. Melo \& I. Alves-dos-Santos, eds.). Editora UNESC, Criciúma, p.297-302.

VELLOSO, A.L., SAMPAIO, E.V.S.B., GIULIETTI, A.M., BARBOSA, M.R.V., CASTRO, A.A.J.F., QUEIROZ, L.P., FERNANDES, A., OREN, D.C., CESTARO, L.A., CARVALHO, A.J.E., PAREYN, F.G.C., SILVA, F.B.R., MIRANDA, E.E., KEEL, S. \& GONDIM, R.S. 2002. Ecorregião da Depressão Sertaneja Setentrional. In Ecorregiões propostas para o Bioma Caatinga (A.L. Velloso, E.V.S.B. Sampaio \& F.G.C. Pareyn, eds.). Flamar Gráfica e Editora, Recife, p.18-21.

VIEIRA, M.F., MEIRA, R.M.S.A., QUEIROZ, L.P. \& MEIRA NETO, J.A.A. 1992. Polinização e reprodução de Jacaranda caroba (Vell.) D.C. (Bignoniaceae) em áreas de cerrado do sudoeste brasileiro. In Anais do 8o Congresso da Sociedade Botânica de São Paulo (SBSP), Fapesp, São Paulo, p.13-19.

WADDINGTON, K.D. 1976. Pollination of Apocynum sibiricum (Apocynaceae) by Lepidoptera. The Southwestern Naturalist 21:31-36.

WADDINGTON, K.D. 1983. Foraging behavior of pollinators. In Pollination biology (L. Real, ed.). Academic Press, Orlando, p.51-86.

WASER, N.M. \& PRICE, M.V. 1991. Reproductive costs of self-pollination in Ipomopsis aggregata (Polemoniaceae): are ovules usurped? American Journal of Botany 78:1036-1043.

YEO, P.F. 1993. Secondary pollen presentation: form, function and evolution. PIant Systematics and Evolution, Supplementum 6, Springer-Verlag, Wien, New York. 